\title{
PROGRESS TOWARD SONIFYING NAPOLEON'S MARCH AND FLUID FLOW SIMULATIONS THROUGH BINAURAL HORIZONS
}

\author{
Dr. Peter W. Coppin \\ Perceptual Artifacts Lab, \\ Faculty of Design and \\ Inclusive Design, \\ Ontario College of Art and \\ Design University, \\ Toronto, Canada \\ pcoppin@faculty.ocadu.ca
}

\author{
Richard C. Windeyer \\ Centre for Drama, Theatre and \\ Performance Studies, \\ Knowledge Media Design Institute, \\ University of Toronto and Perceptual \\ Artifacts Lab, Ontario College of Art \\ and Design University, Toronto, Canada \\ r.windeyer@mail.utoronto.ca
}

\author{
Daniel E. MacDonald \\ Biomedical Simulation \\ Laboratory, Department of \\ Mechanical \& Industrial \\ Engineering, University of \\ Toronto, Toronto, Canada \\ demacdo@mie.utoronto.ca
}

\author{
Dr. David A. Steinman \\ Biomedical Simulation \\ Laboratory, Department of \\ Mechanical \& Industrial \\ Engineering, University of \\ Toronto, Toronto, Canada \\ steinman@mie.utoronto.ca
}

\begin{abstract}
Cross-modal data analytics - that can be rendered for experience through vision, hearing, and touch-poses a fundamental challenge to designers. Non-linguistic sonification is a well-researched means for non-visual pattern recognition but higher density datasets pose a challenge. Because human hearing is optimized for detecting locations on a horizontal plane, our approach recruits this optimization by employing an immersive binaural horizontal plane using auditory icons. Two case studies demonstrate our approach: A sonic translation of a map and a sonic translation of a computational fluid dynamics simulation.
\end{abstract}

\section{CROSS-MODAL DIAGRAMS}

Figure 1a shows some physical material that has been configured in a manner that represents, models, or maps a situation. Carved marks, as notches, are arranged on the material (wood) in geometric, topological, or iconic relations with other marks (notches). The result models, maps, or represents the concrete structure (or shape) of a geographic region.

Recruiting Larkin and Simon's classic definitions [1], this physical arrangement of notches is essentially a diagram, defined as items indexed to a plane. It is certainly not a sentencedefined as items indexed to a sequential list, where each item of the list is only adjacent to the item before and after it on the list - but it also does not rely solely on visual perception. It represents, or models, a situation both visually and tactilely, suggesting cross-modal mappings that transcend visual approaches that dominate contemporary digital media and data analytics research.

Taking this further, there is nothing inherently "visual" about any diagram. As noted, the spatial, topological and geometric properties of a diagram can be mapped to spatial properties of a tactile surface. In addition, they can also be mapped to spatial properties of sound. The items that are indexed to the spatial properties of sound can then be made available to perception via text-to-speech labels, earcons, or auditory icons.

This research was funded by the Canadian Natural Sciences and Engineering Research Council, the Social Sciences and Humanities Research Council, and the Heart \& Stroke Foundation.

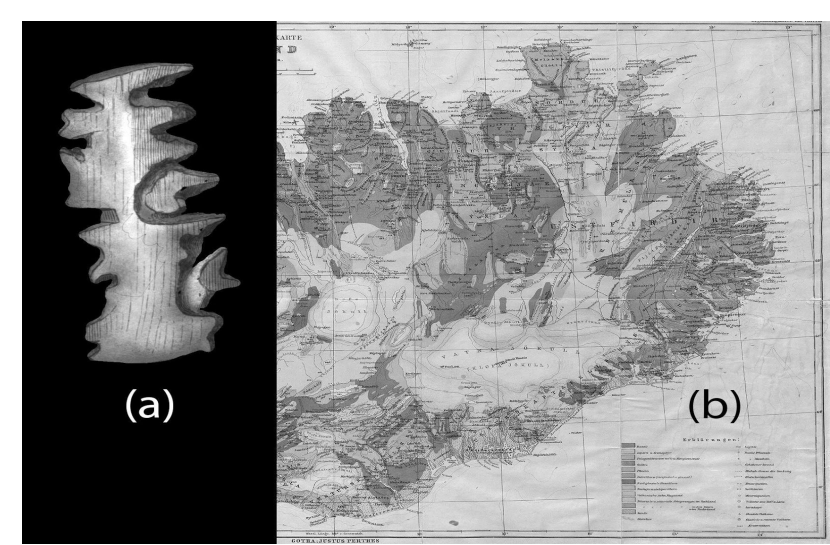

Figure 1: An indigenous diagram, which can be perceived both visually and tactilely (a); in comparison to one of European origin, which is purely visual (b). Adapted from [2, p. 250] and [3]. Public domain.

Although such a mapping from visual to sonic diagrams seems plausible, in practice, obstacles abound, particularly when attempting to construct high-density diagrams - a fundamental challenge faced in virtually all areas of information design. It is in this area of high-density sonification that this paper makes several contributions.

First, we report on our progress toward developing sonic translations of high-density visualizations that are currently accessible only through visual perception through two "grand design challenges": Charles Minard's classic infographic Figurative Map of the Successive Losses in Men of the French Army in the Russian Campaign 1812-1813 (1869) [4][5, p. 40] (Figure 2), and simultaneously, aspects of computational fluid dynamics (CFD) blood flow data in order to augment conventional flow visualizations and increase ease of interpretation of the data.

In addition to reporting on our progress above, we describe how simultaneous development on two distinct design challenges reveal underlying principles and cross-compatible solutions (that underpin both examples), foreshadowing a provisional taxonomy of cross-modal mappings.

Finally, in the process of describing the reasoning behind our design choices, we provide an overview for how work from the ICAD community maps conventions that we are employing.

\section{(i) (5)} Attribution - Non Commercial 4.0 International License.

The full terms of the License are available at http://creativecommons.org/licenses/by-nc/4.0/ 


\section{GRAND DESIGN CHALLENGE: SONIFYING AN EARLY STATISTICAL GRAPHIC}

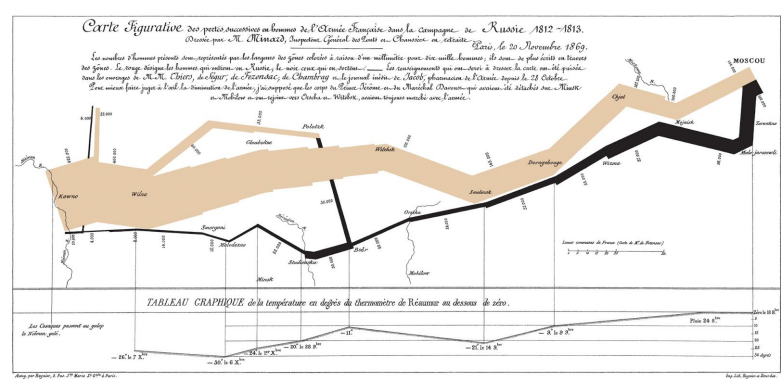

Figure 2: Charles Minard's Figurative Map of the successive losses in men of the French Army in the Russian campaign 1812-1813 (1869). Public domain.

Charles Minard's 1869 infographic is remarkable for its ability to compress six types of data (number of troops; geographical distance; changing temperature; latitude and longitude; travel direction; and location-specific dates) into a two-dimensional representation [4]. The journey undertaken by Napoleon's troops can be decomposed into several variables: Troop Quantity, Troop Position, Troop Direction, and Troop Distance Travelled. A visually perceived line on the rectangular map surface conveyed these variables: The positions of each point of the line conveyed latitude and longitude information (Troop Position), travel direction (Troop Direction), and geographical distance (Troop Distance). A legend in the map used written graphics to show that the line represented the troops' journey. Minard used line thickness to convey Troop Quantity throughout the journey.

\subsection{Selection and design of auditory icons}

Our sonic translation [7] employed sound recordings of marching footsteps (on various outdoor surfaces), seasonal environmental cues (such as birdsong, wind, and snow) to convey changes in terrain and season encountered by the troops, and battle sound effects (such as gunshots and cannon fire to indicate battle locations). The temperature fluctuations that occurred during the army's return were depicted by a tuning frequency of a sine tone.

\subsection{Mapping auditory icons to a horizontal ground plane}

Through the use of an existing dataset extracted from Minard's infographic [8] and Ambisonic spatial encoding techniques [9] within the Max/MSP programming environment [10], our auditory icons were indexed to a horizontal ground plane or "soundmap" (Figure 3). From a fixed listening position at the centre of the map, the changing locations of troops at each point in the journey, their direction, and distance travelled (Troop Position, Direction, and Distance) are conveyed.

Troop Quantity was conveyed at each point in the journey by manipulating a combination of density, pitch/frequency, and tempo of the auditory icon: fewer troop quantities corresponded to the sound of fewer troops marching at a slower tempo and higher pitch, whereas more troops were conveyed by the sound of more troops marching at a faster tempo and lower pitch. Troop Quantity was mapped to multiple sonic parameters simultaneously in order to replicate a key visual feature of Minard's efficient infographic design: the colour-coded layering of the army's advance and retreat trajectories (gold and black respectively), on top of each other.

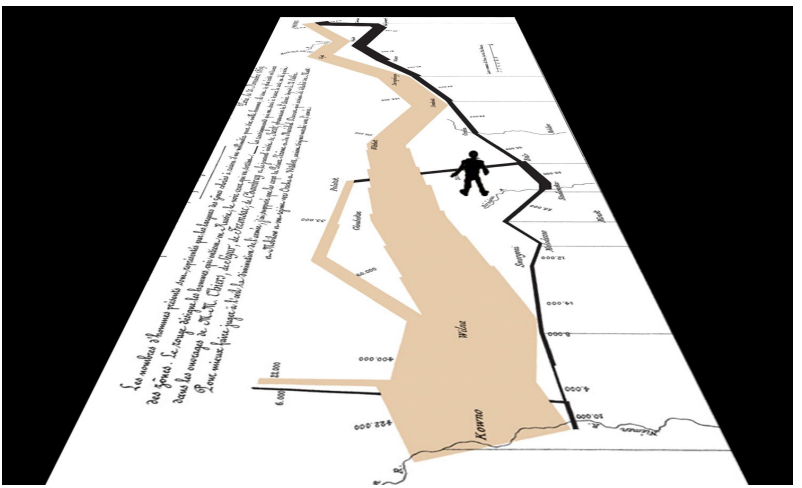

Figure 3: Sketch of the horizontal ground plane or "soundmap."

\subsection{User interaction}

While our initial design iterations supported only linear playback of the sonification, subsequent attempts at facilitating user interactivity produced several working metaphors which continue to inform our work-World of Warcraft and Spot Mic Radar:

1. World of Warcraft (WoW) evokes an immersive and navigable auditory experience of Minard's infographic, with or (ideally) without reliance on its visual presence.

2. Spot Mic Radar describes a more selective means of auditioning the sonification, whereby a user may rotate a virtual microphone around the horizontal ground plane from their fixed listening position at its centre. The user's speed of rotation affects the rate at which the changes in auditory icons may be discerned. At maximum speed, the effect is akin to the rotational detection of objects by a radar system. As with many real-world microphones, the directionality or "pickup pattern" of this "spot mic" is variable. This functions as a variable cone of attention, enabling a user to minimize cacophony by reducing the perceived width of the binaural image from fully binaural to monaural.

\section{GRAND DESIGN CHALLENGE: SONIFYING ANEURYSMAL BLOOD FLOW}

Just as Minard's infographic is remarkable for its ability to compress six types of data into a two-dimensional representation, the power of CFD visualization lies in how it recruits the parallel pattern recognition capability of vision to compress spatial and temporal qualities of numerous types of point data into a three-dimensional representation (two dimensions over time).

Our intention for this second design challenge is to enhance the analytic process of evaluating large datasets by augmenting visual representation through the application of the previously-mentioned sonification methods.

\subsection{Context}

Blood flow patterns are thought to contribute to the development of vascular disease [11][12]. Direct measurement of such flow features is challenged by the limited spatial and/or temporal resolution of current non-invasive medical imaging modalities. Instead, vascular geometries derived from non-invasive medical imaging are used as the basis for "patient-specific" CFD simulations of the blood flow dynamics [13]. 
The motivation of the Biomedical Simulation Lab for sonifying CFD datasets is to introduce a method by which clinicians can characterize recently-uncovered turbulent-like flows in intracranial aneurysms [14], in order to evaluate the likelihood of rupture. Inspired by the natural perceptual encoding between flow regime and sound, a model is proposed whereby the presence and properties of high-energy flow structures within the simulated aneurysmal flow will be communicated via sound.

\subsection{Description of Data}

These simulations result in data structures of high spatial and temporal resolution describing the system hemodynamics in three-dimensional space for a given period of flow [12]. Such results are conventionally inspected using the investigator's choice of interactive scientific visualization applications, such as ParaView [15]. Selection of perspective is required in order to prevent occlusion of important data while balancing the quantitative and qualitative properties of the flow.

By decomposing these volumetric aneurysmal flows in the frequency domain, the flow instabilities can be quantified [16]. Visually communicating these spatiotemporal instabilities ignores the existing strengths of the human auditory system for resolving frequencies as compared to visual perception of spatial frequencies. By sonifying these frequency-based operators, further spatiotemporal characteristics may be uncovered and will be used to augment conventional flow visualizations.

\subsection{Existing techniques}

Several methods of sonifying CFD-generated data have been explored to a limited extent over the last two decades. Klein and Staadt [17] provide methods for analyzing three-dimensional vector fields via sonification. Local flow direction, velocity, and vorticity are conveyed as the observer moves a sphere (the user's "head") through the vector field. Kasakevich et al. [18] furthered the work of Klein and Staadt by detailing methods for mapping flow properties of CFD data to a sonification model with the goal of increasing comprehension. Navigation occurs in a threedimensional space where spatialization techniques are employed.

\subsection{Toward a design solution: selection of auditory icons}

Although our research began independently of the work of both Klein and Staadt and Kasakevich et al., it has converged upon a similar solution in which high-density data sonification is navigated within a three-dimensional auditory field.

Much like visual representation, sonification of this data may benefit from logical grouping; for example, by isolating turbulent-like structures using the methods of Khan et al. [14], pockets of high-energy flow instabilities can be inspected and interpreted much like looking at an isosurface in a visual representation. This requirement for grouping is explored in detail by Klein and Staadt. These volumetric structures may then be sonified based on the size, intensity, and motion. By limiting auditory information within the perceptual field, multiple high-energy pockets may be discerned under interactive inspection while interpreting local patterns and anomalies. The proposed auditory icon solution may resemble sonic forms akin to tornadoes swirling around and past the listener.

\subsection{Prototype: a soundmap for user interaction}

Prototyping began with a search for digital sound synthesis instrument capable of evoking a convincing impression of turbulence in fluid flow when driven by CFD data. Based on a computational model developed and documented by [19], our instrument employs frequency modulation (FM) synthesis in combination with envelope generators, bandpass filters, and physically-modelled tube resonators. Our instrument model was then ported to the SuperCollider programming language [20] in order to increase portability within the lab while easily interfacing with the existing file structures of our CFD data.

Our current prototype [21] situates the user within a three-dimensional virtual auditory space as an extension of our WoW paradigm (Figure 4). The auditory icon allows the local intensity of blood flow to naturally emerge from the velocity trace data. The user may explore a conventional visualization of the flow, where flow-inspired auditory icons are used to indicate the properties of high-energy flow structures. This approach to labelling remained throughout our iterative prototyping.

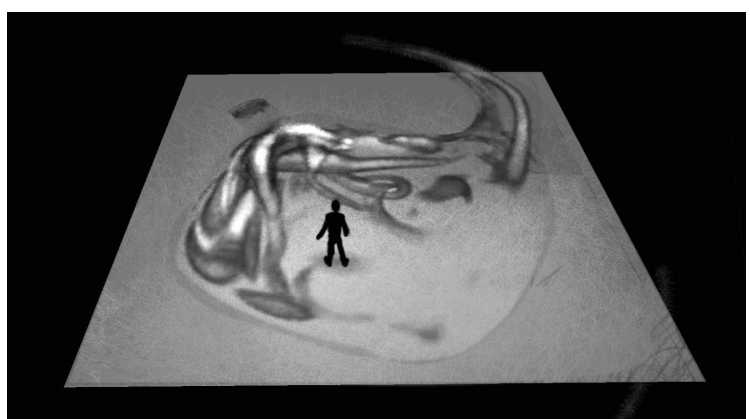

Figure 4: Sketch of a ground plane for representing blood flow trajectories within an aneurysm

\section{DISCUSSION AND IMPLICATIONS}

Let us now consider some underlying principles and mappings that transcend these two design challenges. These principles include i) the use of auditory icons for the labelling of items indexed to a plane and ii) the use of map conventions for limiting the perceptual field and reducing disorientation of the user.

Three techniques were employed in addressing the complexity of our two grand design challenges: the design of auditory icons, the labelling of items indexed to a plane, and the use of a variable cone of attention control to limit the user's perceptual field (our "spot mic radar" paradigm).

\subsection{The labelling of indexed items by auditory icons}

The design of auditory icons allows effective labelling of items indexed to a plane. To label items indexed to a plane in each diagram, auditory icons were positioned within a virtual spatial environment and conveyed via binaural audio.

For our sonic translation of Minard's graphic, we employed the binaurally perceived sound of troops marching along a trajectory as an auditory icon to convey Troop Position, Direction and Distance. Troop Quantity was conveyed by manipulating the auditory icon: fewer troops were conveyed by the sound of fewer troops marching, and vice versa.

Similarly, the intensity of local flow instabilities are conveyed using auditory icons designed to evoke impressions of the chaotic nature of turbulent flowing fluid. Regions of high-energy flow will drive the parameters of our synthesis model, creating a caricature of flow containing a broad range of sonic frequencies with more chaotic change in filter modulation. 


\subsection{Dealing with disorientation while reducing cacophony}

The use of our "spot mic radar" avoids cacophony within a plane of indexed items and reduces disorientation. (Cacophony refers to the ambiguity caused by a number of cues impinging simultaneously.) Our sonic version of a retinotopic map-the World of Warcraft paradigm - avoided cacophony via a narrow detection sphere that avoided signals from both trips simultaneously.

Our blood flow sonification avoids cacophony by enabling the user to selectively choose their position within the flow while adjusting the size and scope of the perceivable auditory field. The use of auditory icons and logically grouped data allows several levels of detail to be presented simultaneously. The end-user design of our flow sonification differs from that of our Minard sonification; the augmentation of flow visualizations allows the user to orient themselves by way of the visual interface. Still, concepts such as the spot mic radar paradigm may be useful in communicating peripheral localization of high-energy flow structures.

\section{CONCLUSION}

This paper reports on two simultaneous grand design challenges with the intention of developing complementary approaches to the sonification of data produced by flow-like structures and dynamics - such as marching soldiers and blood flow. Both projects arrived at cross-compatible solutions that situate a single user within a navigable virtual auditory environment from a first-person perspective. Similarly, both required additional strategies for reducing cacophony by limiting the amount of sonic information present within the perceptual field. In addressing the challenges of sonifying fluid flow data, the natural perceptual encoding of flow regime is employed to convey the presence of unstable turbulent-like structures using auditory icons within a limited perceptual field. Further revisions of these prototypes will refine the proposed cross-modal taxonomy and techniques.

\section{REFERENCES}

[1] Larkin, J. H. \& Simon, H. A. (1987). Why a diagram is (sometimes) worth ten thousand words. Cognitive Science, 11, 65-99. doi:10.1111/j.1551-6708.1987.tb00863.x

[2] Holm, G. \& Garde, V. (1887). Den danske Konebaads-Expedition til Grønlands Østkyst. Retrieved March 6, 2017 from https://books.google.com/

[3] Thoroddsen, T. (1906). Geologische Karte von Island [Map]. Retrieved March 6, 2017 from http://islandskort.is/is/map/show/608

[4] Minard, C. J. (1869). Carte figurative des pertes en hommes de l'Armée Française dans la campagne de Russie 1812-1813 [Infographic]. Retrieved May 15, 2017 from https://commons.wikimedia.org/wiki/File:Minard.png

[5] Tufte, E. R. (2001). The Visual Display of Quantitative Information. Cheshire, CT: Graphics Press.

[6] Corbett, J. (2001). Charles Joseph Minard: Mapping Napoleon's March, 1861. In D. Janelle (Ed.), CSISS Classics. Center for Spatially Integrated Social Science. Retrieved May 15, 2017 from http://escholarship.org/uc/item/4qj8h064

[7] Windeyer, R. C. (2017, May 10). Sonification Prototype: a binaural translation of Charles Minard's infographic
"Figurative Map of the Successive Losses in Men of the French Army in the Russian campaign 1812-1813" (1869) [Video file]. Retrieved May 14, 2017 from https://vimeo.com/217423986

[8] Napoleon's March Data [Computer source code]. (n.d.). Retrieved May 15, 2017 from http://www.datavis.ca/gallery/minard/minwilk.sas

[9] Cycling '74. (n.d.). Max/MSP. Available May 15, 2017 at http://www.cycling74.com

[10] Morbiducci, U., Kok, A. M., Kwak, B. R., Stone, P. H., Steinman, D. A., \& Wentzel, J. J. (2016). Atherosclerosis at arterial bifurcations: evidence for the role of haemodynamics and geometry. Thrombosis and Haemostasis, 115(3), 484-492.

[11] Turjman, A. S., Turjman, F., \& Edelman, E. R. (2014). Role of fluid dynamics and inflammation in intracranial aneurysm formation. Circulation, 129(3):373-82.

[12] Taylor, C. A. \& Steinman, D. A. (2010). Image-based modeling of blood flow and vessel wall dynamics: Applications, methods and future directions. Annals of Biomedical Engineering, 38(3), 1188-203. doi:10.1007/s10439-010-9901-0

[13] Valen-Sendstad, K. \& Steinman, D. A. (2014). Mind the gap: Impact of computational fluid dynamics solution strategy on prediction of intracranial aneurysm hemodynamics and rupture status indicators. American Journal of Neuroradiology, 35, 536-543.

[14] Sandia Corporation \& Kitware. (2005). ParaView [Computer software]. Retrieved May 15, 2017 from http://paraview.org

[15] Khan, M. O., et al. (2017). On the quantification and visualization of transient periodic instabilities in pulsatile flows. Journal of Biomechanics, 52, 179-182. doi:10.1016/j.jbiomech.2016.12.037

[16] Klein, E. \& Staadt, O. G. (2004). Sonification of three-dimensional vector fields. In Proceedings 12th SCS High Performance Computing Symposium (HPC 2004), Society for Modelling and Simulation International, 2004.

[17] Kasakevich, M., Boulanger, P., Bischof, W. F., \& Garcia, M. (2007). Augmentation of visualisation using sonification: A case study in computational fluid dynamics. In IPT-EGVE Symposium, 2007. doi:10.2312/PE/VE2007Short/089-094

[18] Farnell, A. (2010). Designing sound. Cambridge, MA: MIT.

[19] McCartney, J. et al. (n.d.). SuperCollider [Computer software]. Retrieved May 15, 2017 from http://supercollider.github.io

[20] Windeyer, R. C. (2017, May 13) Sonification Prototype: Blood flow caricatures in three-dimensional auditory space [Video file]. Retrieved May 14, 2017 from https://vimeo.com/182031056

\section{ACKNOWLEDGMENTS}

The researchers wish to thank Dr. C. Chnafa and O. Omari (Biomedical Simulation Laboratory, Department of Mechanical and Industrial Engineering, University of Toronto), D. Retren (Perceptual Artifacts Lab, Ontario College of Art and Design University), and A. Li (Perceptual Artifacts Lab, Ontario College of Art and Design University). Peter Coppin would especially like to thank his students R. Vroom and B. Biggs, as well as other students from INCD Lab 1 who worked on the "Sonic Wayfinding Project," for the many conversations that informed the development of the "World of Warcraft" paradigm. 